\title{
La relación entre los creativos independientes de la televisión pública y la audiencia infantil ${ }^{\star}$
}

\author{
Carlos Gutiérrez-González** \\ Recibido: 2019-02-19 • Enviado a pares: 2019-02-25 \\ Aprobado por pares: 2019-03-28 • Aceptado: 2019-05-20 \\ https://doi.org/10.22395/angr.v18n35a3
}

\begin{abstract}
Resumen
Este artículo explica cómo se presenta la relación entre los directores, guionistas, productores, realizadores e investigadores independientes de la televisión pública de Chile, Colombia y México, y la audiencia infantil; además, establece los criterios para definir un público objetivo. Para este estudio de corte cualitativo, se aplicaron 21 cuestionarios a los realizadores de programas emitidos a través de los canales de televisión pública como TVN y Novasur de Chile, el canal Once y Canal 22 de México y Señal Colombia, los cuales recibieron nominaciones o premios nacionales e internacionales desde su emisión. Los resultados evidencian que la relación se presenta por iniciativa propia y no por los canales. Además, esta se produce tanto en la fase creativa del proyecto - para testear su viabilidad - como en la etapa de producción de los contenidos durante las grabaciones. Sin embargo, se identifica que no hay un seguimiento de la aceptación y apropiación de los mensajes producidos y emitidos por los canales de televisión, especialmente en aquellos que producen propuestas culturales y de eduentretenimiento - uno de los objetivos de la televisión pública-. Finalmente, los creativos de la televisión brindan una serie de recomendaciones importantes para diseñar proyectos audiovisuales infantiles, que tienen en cuenta aspectos como la investigación, contenidos, producción y participación de la audiencia.
\end{abstract}

Palabras clave: audiencia; infancia; producción televisiva; productor de radio/TV; interacción social; sistema de clasificación; proceso de comunicación; contenido de programa.

Este artículo es resultado del proceso de formación doctoral en Comunicación de la Universidad del Norte, el cual se financió con recursos del Fondo Nacional de Financiamiento para la Ciencia, la Tecnología e Innovación FcTEI Del Sistema General de Regalías (SGR).

* Doctorando en Comunicación de la Universidad del Norte, Barranquilla, Colombia. Productor de televisión y profesor asistente de la Facultad de Educación en la Fundación Universitaria del Área Andina, Bogotá, Colombia. Correo electrónico: cgutierrez@areandina.edu.co. Orcid: https://orcid.org/00000002-0646-4653 


\title{
The Relationship Between Independent creatives of Public Television and Child Audience
}

\begin{abstract}
This paper explains how the relationship among independent directors, producers, screenwriters, and researchers of public television from Chile, Colombia, Mexico and child audiences is established, it also sets the criteria to define the target group. For this qualitative study, 21 questionnaires were applied to creatives of broadcasted programs of public television channels like TVN and Novasur from Chile, Canal Once and Canal 22 from Mexico, and Señal Colombia, all of which received nominations or national and international awards since their emissions. The results show that the relation is present by own initiative and not by the channels. Moreover, this is conformed in the same project creation phase to test its viability and in the production stage of contents during recordings. Nevertheless, it is identified that there is no follow-up about the acceptation and appropriation of produced and broadcasted messages by television channels, specially those that produce cultural edu-entertainment shows, as it is one of the objectives of public television. Finally, television creatives give a series of important recommendations for designing a children's audiovisual project, taking into account aspects such as research, contents, production, and audience participation.
\end{abstract}

Keywords: audience; childhood; television production; radio/TV producers; social interaction; classification systems; communication process; programme content.

\section{A relação entre os criativos independentes da televisão pública e a audiência infantil}

\begin{abstract}
Resumo
Este artigo explica como se dá a relação entre os diretores, roteiristas, produtores, realizadores e pesquisadores independentes da televisão pública do Chile, da Colômbia e do México, e a audiência infantil. Além disso, estabelece os critérios para definir um público-alvo. Para este estudo de corte qualitativo, 21 questionários foram aplicados aos realizadores de programas emitidos por meio dos canais de televisão pública como TVN e Novasur do Chile, o canal Once e Canal 22 do México e Señal Colombia, que receberam nominações ou prêmios nacionais e internacionais desde sua transmissão. Os resultados evidenciam que a relação se dá por iniciativa própria e não pelos canais. Ainda, esta é produzida tanto na fase criativa do projeto para testar sua viabilidade quanto na etapa de produção dos conteúdos durante as gravações. No entanto, identifica-se que não há um acompanhamento sobre a aceitação e apropriação das mensagens produzidas e emitidas pelos canais de televisão, especialmente naqueles que produzem propostas culturais e de entretenimento educativo - um dos objetivos da televisão pública. Finalmente, os criativos da televisão oferecem uma série de recomendações importantes para o momento de criar um projeto audiovisual infantil, que leva em consideração aspectos como a pesquisa, os conteúdos, a produção e a participação da audiência.
\end{abstract}

Palavras-chave: audiência; infância; produção televisiva; produtor de rádio/TV; interação social; sistema de classificação; processo de comunicação; conteúdo de programa. 


\section{Introducción}

Las audiencias, en especial la infantil, son objeto de estudio permanente por parte de los científicos sociales. Sus objetivos se han centrado en analizar variables como clase, género, edad, circunstancias familiares u origen étnico (Hartley, 2002); indagar por los efectos que producen los medios (Ortega, Pereira, e Igartua, 2016; Pearce y Field, 2016), su transformación con el desarrollo de las plataformas sociales digitales (Hallvard, Poell y van Dijck, 2015); e incluso cuestionar si han dejado de existir (Livingstone y Das, 2013).

De acuerdo con Livingstone y Das (2013), la importancia de los estudios de audiencia radica en los contenidos, con énfasis en su "lectura, escritura, interpretación y las consecuencias de estas prácticas para la inclusión o exclusión, aceptación o crítica" (p. 105). En ese sentido, se podría manifestar que la escritura es una labor que compete a quien produce el mensaje; mientras que la recepción, lectura e interpretación serían funciones de la audiencia. Sin embargo, estos procesos tendrían como objetivo una construcción de sentido a partir de una relación de doble vía en la cual destaque una interacción directa y dialógica entre el productor del contenido y la audiencia (Hall, 2012).

Con este planteamiento, y después de haber realizado un barrido por bases de datos especializadas como Ebsco, Scopus y Web of Science — en las cuales se revisaron artículos publicados entre 2010 y 2017-, se evidencia que más allá del protagonismo que tienen en la co-creación de mensajes publicitarios (Fernándes y Remelhe, 2016) o el poder que han adquirido las comunidades de fans (Hadas y Shifman, 2013), los productores, directores y libretistas de la televisión no han sido protagonistas habituales de los estudios de audiencias en tanto creadores de contenidos audiovisuales. Es decir, la posible relación directa con su comunidad imaginada (Hartley, 2002), especialmente con las audiencias infantiles, no ha sido considerada por los investigadores como un aspecto relevante en sus procesos empíricos, ya que "los estudios de audiencia y los estudios de producción han tenido trayectorias separadas a pesar de compartir la agenda de la teoría fundamentada y los métodos de investigación" (Mayer, 2016, p. 1).

\section{La comunidad imaginada}

El término 'audiencia' se ha empleado para definir al grupo de personas que usan los medios de comunicación (Hartley, 2002) — puntualmente, radio y televisión-. Además, se emplean los sustantivos 'masa' y 'mercado' para hacer referencia al colectivo de personas que acceden a sus transmisiones (Livingstone y Das, 2013). Asimismo, y debido a su diversidad demográfica, este concepto se utiliza para describir un gran número de personas no identificables, lo que generalmente implica "homogeneizarla[s], atribuyéndole[s] ciertas características, necesidades, deseos y preocupaciones" (Hartley, 2002, p. 11). 
Livingstone (2005) señala que las audiencias, tanto en las élites como en los discursos populares, reciben la calificación de triviales, pasivas e individualizadas; cosa distinta ocurre con los públicos, que se valoran como activos, críticos y políticamente significativos. De igual manera, Livingstone (2005, citando a los precursores de la Escuela de Frankfurt) manifiesta que este enmarque tiene una relación directa con los intereses económicos, políticos, estéticos, ideológicos y profesionales de los medios; los imperativos competitivos y las obligaciones comerciales que orientan a los productores de contenidos; la polisemia de los textos; la falta de mapeo simple entre el contenido textual y la recepción de la audiencia, porque los contextos de la codificación y decodificación difieren sistemáticamente; y con la tensión en el desarrollo tecnológico entre la tendencia hacia la centralización —estandarización-y la diversificación —innovación-.

En la actualidad, las audiencias de la televisión,

(...) no solo se han dividido en grupos cada vez más reducidos, por una mayor oferta de canales disponibles, con perfiles y oferta atractivos para grupos bastante más acotados; también se han fragmentado por un aumento relevante de dispositivos para consumir televisión. (Buzeta y Moyano, 2013, p. 56)

Dentro de esa variedad de contenidos televisivos se encuentran los canales especializados para niños: además de disponer su sistema de transmisión en señal abierta o en las parrillas de programación de los operadores por cable y satelitales, estos han ampliado y fortalecido sus sitios en internet de forma continuada, con recursos interactivos de entretenimiento como juegos online y offline. De esta manera, los medios de comunicación están adaptando sus contenidos a las demandas de una audiencia multipantalla e hiperconectada, vinculada al ocio y al consumo con dispositivos cada vez más interactivos e inteligentes (Cáceres y Brändle, 2011; Ortega, González Ispierto y Pérez Peláez, 2015).

En los estudios de audiencia se suelen incluir consumos de televisión a escala de hogar y de individuos caracterizados por edad desde los cuatro años, sexo, nivel socioeconómico, acceso a la televisión de pago (Buzeta y Moyano, 2013). Ahora, no solo se recomienda la investigación cuantitativa que define sistemas de medición como el rating o el share, sino que se plantea la necesidad de estudiar las emociones a partir de métodos neurocientíficos cualitativos (Crespo-Pereira, Martínez-Fernández y Campos-Freire, 2017).

La edad ha sido una variable fundamental para segmentar las audiencias infantiles. En los estándares de la televisión internacional, se considera la siguiente clasificación: TV-Y corresponde a los programas para niños entre 2 y 6 años; TV-Y7 para niños mayores de 7 años; y TV-G para todas las edades (Federal Communications Commission, 2016). No obstante, Nielsen Media Research y otras compañías de investigación han 
tenido como punto de partida dos rangos de edad diferentes para la audiencia infantil: niños de 2 a 5 años y entre los 6 y los 11 años de edad (Cahm, Kalagian, y Lyon, 2008).

En ese orden de ideas, Hartley (2002) comenta que las audiencias

[...] permiten a las organizaciones de medios vender publicidad o cumplir con sus obligaciones públicas y estatutarias, [por lo que] es importante conocer el tamaño, la calidad, composición demográfica y las características de las audiencias, $[\ldots]$ datos que se relacionan directamente con los ingresos. (p. 12)

Ante la situación planteada, los anunciantes, agencias de publicidad, agencias de prensa o proveedores de investigación de mercados han sido los más interesados en conocer en detalle la caracterización de las audiencias (Esomar, 2009). Entonces, en una cadena de producción, y puntualmente en la referida a la industria de la televisión privada en la cual los ingresos económicos ocupan un lugar relevante, los productores acceden a información concerniente a los estudios de factibilidad de los proyectos a desarrollar. Entonces, ¿̇qué ocurre con aquellos creativos independientes de la televisión pública que gestionan recursos del Estado para desarrollar proyectos audiovisuales? ¿Cómo se las ingenian para obtener los informes necesarios para garantizar el primer paso del diseño creativo de un proyecto? ¿Lo hacen? ¿Es necesario para ellos conocer e interactuar con las audiencias?

Como se puede evidenciar, son muchas las preguntas que surgen en la relación entre los creativos de la industria audiovisual y las audiencias. A continuación, se citan los estudios más próximos, realizados entre 2010 y 2017, que involucran la interacción entre productores y audiencia, pero siempre en una sola vía y no como sujetos que dialoguen entre sí. En primera instancia, Sekarasih, Nayar, O'Malley, Olson, y Scharrer (2016) realizaron un análisis cualitativo de 35 cartas enviadas a un programa de alfabetización mediática que interpretan estudiantes entre los 10 y 11 años de edad de la región de Nueva Inglaterra (Estados Unidos). Además, los investigadores incluyeron la opinión de los niños con respecto a la responsabilidad de los productores de contenido, quienes — según los menores - interpretan a los infantes como consumidores y no como ciudadanos.

Por su parte, Zanker (2011) examinó los discursos infantiles de la producción televisiva del canal público educativo Weta de Nueva Zelanda. Su pregunta de investigación fue ¿̇cómo imaginan los productores a sus audiencias y construyen espacios públicos apropiados para ellos dentro de las restricciones actuales de la regulación de la financiación y la publicidad? El trabajo de campo se desarrolló en 2009, con entrevistas cara a cara realizadas a los productores. En los resultados se expone que los productores hacen las veces de "abogados" de los niños para darles la oportunidad de participar en los diferentes espacios de la programación. 
Para finalizar este apartado, Martinez (2016) analiza cómo los productores de publicidad construyen la audiencia infantil como una audiencia publicitaria. La investigadora, al aplicar 18 entrevistas a productores suecos, obtiene como resultado que los niños son vulnerables y dependientes. De igual manera, Gozansky (2017) en Israel, mediante la aplicación de las teorías culturales de Bourdieu, realiza 20 entrevistas cualitativas a la misma cantidad de productores que han trabajado en los principales canales de televisión para niños de ese país. Al examinar los cambios significativos que ha tenido la producción de contenidos infantiles desde 1966 al 2010, concluye que los medios audiovisuales fortalecen el concepto de niñez como consumidores.

En América Latina se encuentra la investigación de Vega y Lafaurie (2013), quienes presentan los resultados del observatorio infantil de televisión, Observar TV, en el que, mediante la investigación-acción participativa, los niños socializan sus experiencias y asumen "el reto de discutir, transformar o reafirmar sus imaginarios de ciudadanía" (p. 145), mostrándose críticos hacia la televisión.

De acuerdo con lo expuesto, las preguntas de investigación planteadas para este estudio son las siguientes:

- ¿Cómo se presenta la interacción entre los directores, productores y libretistas de la televisión pública de Chile, Colombia y México, y la audiencia infantil?

- ¿Cuáles son los criterios para definir la composición de la audiencia infantil de los contenidos que producen los creativos de la industria audiovisual?

Por lo anterior, los objetivos de este artículo son identificar la relación de los creativos independientes de la televisión pública infantil chilena, colombiana y mexicana con su audiencia y establecer los criterios de segmentación de la misma. De igual manera, resulta oportuno mencionar que esta investigación contribuye a los conocimientos de los directores, productores, realizadores y libretistas de la industria audiovisual —en especial, de la televisión infantil—, así como a los académicos interesados en los estudios de medios, la alfabetización de medios y el eduentretenimiento, y a los estudiantes de comunicación, cine, televisión y producción audiovisual de pregrado y posgrado.

\section{Metodología}

Para dar respuesta a estas preguntas de investigación, se diseñó un cuestionario en línea con preguntas abiertas y cerradas, dirigido a los creativos independientes de la televisión pública dedicados a la producción de contenidos infantiles en Chile, Colombia y México. Inicialmente, se realizó una búsqueda en internet con los programas infantiles producidos y emitidos entre 2010 y 2017 en los canales públicos TVN y Novasur de Chile, Canal 22 y Canal Once de México, y Señal Colombia, cuyo carácter 
representativo se asignó de acuerdo con la cantidad de nominaciones y premios nacionales e internacionales que han recibido sus contenidos después de emitirse en eventos como el Lápiz de Acero, los Premios India Catalina, el Prix Jeunesse, los Premios TAL, los Premios de Viña del Mar o los Emmy Awards. Entre ellos se encuentran La lleva (González, 2010), Mr. Trance (Cabrera y Veneras, 2013), Guillermina y Candelario (Rincón, Rincón, y de Jesús, 2010), Puerto papel (Ceppi y Covarrubias, 2016), 31 minutos (Díaz y Peirano, 2014), ¿Con qué sueñas? (Gómez, 2010), Mi lugar (Martínez, 2016), Kiplata (Salazar, 2012), Tikitiklip (Egaña y Puga, 2010), Camusi camusi (Santamaría, 2013), El mundo animal de Max Rodríguez (Sánchez, 2013), Pichintún (Veloso, 2015) y Fuimos colores (Almada, 2014).

Luego se procedió a buscar la ficha técnica de cada programa para identificar los nombres de directores, guionistas, productores, realizadores e investigadores, con esa información se elaboró una base de datos con 34 creativos de la industria, fundamentada en los datos registrados en sitios web y en las redes sociales como Facebook. En total, once colombianos, seis mexicanos y cuatro chilenos dieron respuesta al cuestionario virtual, diseñado en la plataforma gratuita de formularios de Google Drive. El cuestionario se dividió en tres secciones. La primera correspondió a la identificación personal de cada productor. En la segunda, se indagó por la relación entre el creativo y su audiencia, con preguntas como "¿ha existido una relación directa entre usted y su audiencia?", "¿cómo podría describir esa interacción con su audiencia?", "¿qué tipo de conclusiones, acuerdos o interpretaciones sobre los contenidos surgen del proceso de interacción con la audiencia?" y "¿quién o quiénes promueven la iniciativa para facilitar esa interacción?"

La última sección planteó preguntas para definir la composición de la audiencia infantil; los esfuerzos se enfocaron en identificar la importancia que el productor da a variables como sexo, edad, estilo de vida, etnia y raza, nivel socioeconómico y ubicación geográfica, para caracterizar u homogeneizar su audiencia al momento de crear un contenido infantil. Finalmente, los productores, realizadores y directores brindaron algunas recomendaciones que se deberían considerar al diseñar y producir proyectos infantiles. Una vez elaborado el instrumento, se procedió a validarlo con un investigador experto en la formulación de este tipo de cuestionarios y con un productor colombiano de televisión infantil.

\section{Resultados}

A continuación se exponen los resultados obtenidos de los cuestionarios: en principio, se describen los momentos en los que se establece la interacción entre realizadores y audiencia, así como sus características; luego se establecen las conclusiones, acuerdos e interpretaciones que propician esa interacción, y se definen los sujetos que promueven ese contacto; por último, se determina la composición de la audiencia infantil 
para los creativos y se cierra con unas recomendaciones que brindan los sujetos de estudio al diseñar un proyecto audiovisual infantil, tomándose en cuenta categorías como investigación, contenidos, producción y participación de la audiencia.

\section{La interacción entre creativos y audiencia}

Todos los creativos de la industria que participaron en el estudio manifestaron que durante la producción y realización de contenidos audiovisuales infantiles han tenido algún tipo de contacto con la audiencia, principalmente en la etapa de investigación (esto es, al iniciar con la propuesta creativa de un nuevo formato) y, en menor medida, luego de la emisión, cuando exhibieron los contenidos en festivales, talleres, muestras audiovisuales o eventos educativos.

Edna Katerine Moreno, realizadora audiovisual colombiana, comentó que su

metodología de trabajo determina hacer investigación directa con la audiencia previa a la formulación de los formatos y con el objetivo de generar un diagnóstico temático y de intereses de la audiencia. Una vez el formato se produce en su fase piloto, se hacen grupos focales con la audiencia en la perspectiva de analizar junto con los chicos su parecer y percepción sobre el producto. (cuestionario en línea, comunicación personal, 7 de junio de 2017)

En ese sentido, los realizadores consideran importante tener contacto con la audiencia para adelantar la investigación de contenidos y evaluar los productos a través de grupos focales, con el fin de enriquecer y consolidar la propuesta creativa. Yenny Santamaría, realizadora de cine y televisión colombiana, afirmó que el contacto directo con la audiencia se ha presentado mediante un "focus group de muestra audiovisual, un focus group del capítulo 1 de una serie, un visionado de productos terminados y pruebas de uso de productos interactivos" (cuestionario en línea, comunicación personal, 25 de mayo del 2017). En cambio, la socióloga mexicana Olga Durón Viveros realiza este contacto en la fase de investigación de contenidos a través de talleres de producción con menores, en los que ellos definen los temas a partir de sus derechos, el acoso escolar, maltrato infantil o violencia familiar. Luego, los participantes realizan los guiones, dibujos y voces para después transformarse en la audiencia (cuestionario en línea, comunicación personal, 27 de mayo del 2017).

De igual manera, algunos creativos reconocieron que el contacto directo con la audiencia infantil ha sido poco y se ha presentado a través de las redes sociales digitales - especialmente en Facebook-, con televidentes que escriben sus opiniones una vez observan el programa al aire. Así lo comenta Maritza Sánchez, directora y guionista colombiana: "el contacto casi nunca ha sido directamente. Casi siempre la interacción es por redes sociales después de la producción" (cuestionario en línea, comunicación personal, 25 de mayo del 2017). 


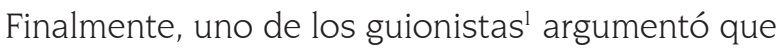

la interacción a través de redes [sociales digitales] es valiosa tanto para conocer opiniones como para mantener el enganche de una audiencia que ya se ha conectado emocionalmente con el contenido, ya que si han escrito es porque les importa el contenido. (cuestionario en línea, comunicación personal, 30 de mayo del 2017)

Los realizadores y productores de televisión infantil describieron la relación con su audiencia con las siguientes palabras clave: limitada, provechosa, interesante, divertida, relativa, enriquecedora, reveladora, cordial, feliz, acogedora, tranquila y horizontal. Para Marcos Almada Rivero, escritor e ilustrador mexicano, la interacción se presenta durante y después de las proyecciones donde "suele haber buena aceptación e interés. Allí, generalmente son los padres los que interactúan, más que los niños. Les llama mucho la atención ver obras con un enfoque local y diferentes al contenido estadounidense" (cuestionario en línea, comunicación personal, 26 de mayo del 2017).

Asimismo, la comunicadora mexicana Cruz Melissa Angulo comentó que en esa interacción lo que se busca es que la audiencia participe y "se sientan escuchados y cómodos" (cuestionario en línea, comunicación personal, 28 de mayo del 2017). Mientras que, Carla Higueras, investigadora y productora periodística chilena, describe la interlocución con la audiencia como un espacio directo y generalmente cordial, de "gran ayuda a la hora de rescatar localidades, historias y personajes" (cuestionario en línea, comunicación personal, 6 de junio del 2017).

Por su parte, Marcela Rincón, directora y guionista colombiana, mencionó que en cada etapa de la producción

[...] buscamos cosas distintas, por ejemplo, en la elaboración del guion testeamos las historias e incorporamos sus sugerencias. En la producción, vemos cómo funcionan los contenidos y si hay algo que podemos mejorar, lo hacemos inmediatamente. Al finalizar, hacemos un visionaje de los contenidos con los niños para entender las cosas que gustan y las que no, con el fin de tenerlas en cuenta en futuras producciones. (cuestionario en línea, comunicación personal, 1 de junio del 2017)

De igual forma, Maritza Rincón, productora colombiana, refuerza la idea anterior, al manifestar que realizan técnicas de investigación como el:

[...] focus group con niños de distintas edades y estratos sociales para evaluar los resultados de nuestras producciones e identificar qué les gusta más, qué les interesa y qué cosas se pierden. Siempre aprovechamos para preguntar qué les gustaría ver en una próxima oportunidad. (cuestionario en línea, comunicación personal, 11 de junio del 2017)

Al responder el cuestionario solicitó que su nombre no fuera citado en ninguna publicación. 
La descripción del proceso de interacción entre creativos de la industria y la audiencia infantil es un "intercambio de saberes, sentires y formas de ver el mundo", como lo comentó el realizador audiovisual colombiano, Wilmer Sotto (cuestionario en línea, comunicación personal, 24 de mayo del 2017), en el cual cada productor busca conocer los gustos e intereses, así como la reacción y aceptación de los niños y niñas a los contenidos.

\section{Conclusiones, acuerdos o interpretaciones de la interacción}

Las respuestas de los realizadores y productores de contenidos audiovisuales infantiles al preguntarles por las conclusiones, acuerdos o interpretaciones que se han presentado en ese proceso de interacción, en su mayoría, estuvieron encaminados al aporte significativo que tiene la audiencia en el diseño creativo del formato a producir. Para ilustrar esto, Yenny Santamaría comentó que el resultado de ese proceso de interacción es la "validación del diseño de personajes, así como la necesidad de ajustes del contenido cuando no es claro para la audiencia y la identificación de elementos a destacar y/o repetir en producciones subsiguientes" (cuestionario en línea, comunicación personal, 25 de mayo del 2017).

En esa misma línea, Sotto concluyó que,

[...] la evidencia del comportamiento de individuos de edades similares a mi público objetivo se convierte en un recurso muy importante para construir el formato audiovisual, el tipo de narrativas a utilizar y cómo desarrollar los temas de mi propuesta audiovisual. (cuestionario en línea, comunicación personal, 24 de mayo de 2017)

Finalmente, Mónica Baquero, directora y guionista colombiana, afirmó que usualmente en los encuentros "aparecen nuevos temas que de pronto no habían sido previstos inicialmente" (cuestionario en línea, comunicación personal, junio 9 del 2017), mientras que el comunicador audiovisual chileno, Patricio Veloso, mencionó que "el humor es un gran catalizador de los mensajes educativos" (cuestionario en línea, comunicación personal, 7 de junio del 2017).

En otro orden de ideas, el diseñador mexicano, Eugenio Valentín Aranda, concluyó que había subestimado el conocimiento y la capacidad de interpretación de contenidos de la audiencia infantil (cuestionario en línea, comunicación personal, mayo 27 del 2017). Y para Marcos Almada, la conclusión más importante fue que los niños y niñas mostraron "emoción al saber que en su país se elaboran materiales originales y con un enfoque en su propio contexto, y hacen comentarios más puntuales sobre la historia o los personajes" (cuestionario en línea, comunicación personal, 26 de mayo del 2017).

Finalmente, Carla Higueras y Julián Gómez, realizador de cine y televisión colombiano, relacionaron a los niños con los adultos. Por un lado, Carla concluyó que 
[...] los niños son mucho más abiertos que los adultos a la hora de hablar de sus problemas, por ejemplo de situaciones que los afectan, como la separación de los padres, falta de recursos económicos, sueños o proyecciones. Ellos se dan cuenta que no son los únicos niños que atraviesan por esas experiencias y se dan cuenta que a pesar de los problemas pueden ser felices como los niños que mostramos en el programa. (cuestionario en línea, comunicación personal, 6 de junio del 2017)

Por su parte, Gómez sentenció que los infantes "quieren ver todo lo que los adultos no queremos que vean" (cuestionario en línea, comunicación personal, 6 de junio del 2017).

\section{Agentes que promueven la interacción}

En el cuestionario se establecieron las siguientes opciones para dar respuesta múltiple a la pregunta ¿quiénes son los agentes que promueven la interacción entre creativos y audiencia?: ¿El canal de televisión, usted o un integrante del equipo de producción, un televidente, un colegio o una universidad, una entidad cultural u otro? Como resultado se obtuvo que ocho de los 21 creativos manifestaron que los canales de televisión han sido los agentes que promueven el ejercicio de interacción entre ellos y la audiencia, mientras que 16 dijeron que ellos mismos son los promotores de estos encuentros, tres mencionaron que una entidad cultural, dos han tenido la experiencia de ser invitados por un colegio o una universidad y, por último, un televidente chileno y otro colombiano convocaron a los creativos a llevar a cabo un ejercicio de interacción.

Al respecto, una realizadora ${ }^{2}$ comentó que ha sido invitada junto con su equipo de producción, para exhibir sus "programas en seminarios organizados por universidades y también por televisiones de otros países y por entidades culturales. En esas ocasiones se ha invitado a niños, pero no hay una evaluación final de su parte. Es solo una muestra" (cuestionario en línea, comunicación personal, 6 de junio de 2017).

\section{Composición de la audiencia infantil}

Los creativos de la industria audiovisual infantil consideran importante segmentar su audiencia por rango de edad, zona geográfica y estilo de vida, no así por nivel socioeconómico ni mucho menos por su etnia o raza, ya que son criterios excluyentes. Al respecto, Edna Katerine Moreno señala que:

Todos los niños del mundo tienen las mismas necesidades cognitivas, emocionales y familiares, lo que sí cambian son sus necesidades sociales, determinadas especialmente por la influencia cultural. Pero a la hora de hacer contenidos de calidad para niños debemos considerar que, en términos de necesidades, son un grupo homogéneo, pero que la creación de las historias

\footnotetext{
Al responder el cuestionario solicitó que su nombre no fuera citado en ninguna publicación.
} 
y la definición de personajes sí pasa por un diseño que atiende a especificidades para poder ofrecer una mirada del mundo que sea específica y a la vez universal. No diseñamos contenidos para niños guambianos o palenqueros o mestizos de Bucaramanga, diseñamos para los niños que según su etapa de desarrollo necesitan esto o aquello y les ofrecemos contenidos sobre niños con los que se ven identificados por la profundidad de su ser, no porque se vistan o vivan de una manera u otra. (cuestionario en línea, comunicación personal, 7 de junio del 2017)

Finalmente, los productores suelen acudir a teorías como las etapas del desarrollo cognitivo de Piaget, o bien a libros de literatura infantil para desarrollar sus proyectos creativos. No obstante, prefieren y ven más enriquecedor poner a disposición su propia experiencia, así como la de otros referentes como Jan Willem-Bult (director, guionista y productor holandés de varios proyectos televisivos y cinematográficos) o acudir a los comentarios publicados en las redes sociales con respecto a los contenidos infantiles. Cabe agregar que, en ocasiones, la información que proveen los canales es relevante en el diseño creativo. Sin embargo, una de las realizadoras manifiesta que muchas veces se deduce el tipo de público por los contenidos que está emitiendo el canal de televisión, mas no porque se les brinde esa información.

\section{Recomendaciones de los productores}

Los realizadores de televisión infantil coinciden en algunas recomendaciones para aportar a un segmento al que se dirige este estudio — sus colegas y estudiantes en formación- para cuando diseñen proyectos dirigidos a infantes. Estas recomendaciones se agruparon en las siguientes categorías:

- Investigación: realizar una investigación sólida es una condición innegociable del equipo creativo de un proyecto audiovisual. Para lograrlo, es necesario conocer el formato, el género, los aspectos culturales y las tendencias de consumo del público objetivo al cual se dirigirá el contenido.

- Contenido: una vez realizada la investigación, el punto de partida debe ser contar historias significativas e inspiradoras para los niños, cargadas de identidad, que muestren la realidad con formas innovadoras, buscar temas de interés y narrar de manera responsable y respetuosa. Además, los creativos recomiendan ser originales en la escritura y el tratamiento audiovisual, sin dejar de lado la premisa de divertir a través del humor. Por último, sugieren crear lazos afectivos entre la historia y los personajes con los menores.

- Producción: dos aspectos clave son el presupuesto y los equipos de trabajo. Por un lado, se debe conocer el financiamiento del proyecto y con cuánto presupuesto se contará para que la investigación y la producción del contenido sea viable; por 
el otro, quienes integran el equipo deberían ser personas sensibles y motivadas al abordar temáticas de la infancia y, preferiblemente, con buen sentido del humor. Finalmente, algunos realizadores plantean la necesidad de hallar una metodología para el trabajo de campo que no agote a los menores.

- Participación de la audiencia: el diálogo con los niños es un punto que resaltan quienes participaron en este estudio - tanto en la etapa temprana del proceso como durante la producción del proyecto - con el fin de validar el contenido, hecho que brinda la posibilidad de construir ideas en conjunto. De igual forma, los contenidos multipantalla son relevantes en esta categoría puesto que se facilita la interacción a través de herramientas tecnológicas (en redes sociales, páginas web o aplicaciones).

Lo anterior no solo es aplicable en los países de origen de las personas que hicieron parte de estudio; son recomendaciones que se adaptan a cualquier país y cultura en los que se pretenda llevar a cabo un proyecto audiovisual infantil.

\section{Discusión y conclusión}

Con la información recogida se establece que existe una relación directa entre los creativos de la industria audiovisual que hicieron parte de este estudio y la audiencia, pero esta es limitada en términos de la transcendencia del infante. Por lo tanto, se puede interpretar que la prioridad en esta interacción es el hecho de considerar a los niños como consumidores y, en menor medida, como sujetos de participación activa con sentido crítico y argumentativo (Gozansky, 2017; Vega y Lafaurie, 2013). Durante este contacto, que generalmente se presenta en la etapa de investigación a través de la técnica del grupo focal (cuya finalidad es validar y testear un contenido que se encuentra en proceso de diseño), se construyen significados que dan viabilidad a un proceso de codificación y decodificación natural del mensaje por parte de la audiencia, como lo manifiesta Hall (2012); sin embargo, no es recomendable generalizar los resultados de grupos pequeños.

Más allá de brindar señales participativas durante la emisión del contenido que brinden a los niños la oportunidad de participar y ensayar contenidos programáticos de manera mecánica o lingüística (Piotrowski, 2014), la otra etapa en la que los productores tienen contacto con su audiencia corresponde a la exhibición del contenido en muestras audiovisuales; empero, ninguno mencionó la importancia de realizar un seguimiento a la aceptación y apropiación de los mensajes emitidos (en especial aquellos creativos que producen propuestas culturales y de eduentretenimiento). Por lo tanto, es relevante que los productores independientes de la televisión pública hagan las veces de abogados, como lo sugiere Zanker (2011), y diseñen estrategias de seguimiento para evaluar el propósito de educación y entretenimiento planteados desde 
la propuesta creativa, a través de propuestas como la epistemología de la visualidad (Ávila y Acosta, 2016). Claro está, para ello se requiere de un mayor compromiso de los canales de televisión pública. A lo anterior se puede agregar que los productores son los facilitadores principales para propiciar el encuentro con su audiencia. Los canales de televisión, por su parte, tendrían una responsabilidad mayor en temas de presupuesto, tiempo, disponibilidad de talento humano o promotores de iniciativas para incluir este aspecto en las convocatorias dirigidas a los creativos independientes. Empero, lo más representativo sería que los canales de televisión brindaran estas posibilidades y destinaran recursos económicos suficientes para conocer mejor a la audiencia infantil en cuanto a gustos, tendencias, rangos de edad y zona geográfica, como se estableció en la composición de la audiencia. De esta manera, no se dejaría a la intuición de los realizadores la búsqueda de respuestas que se hallan con estudios formales, a la vez que se podría indagar si el formato emitido presenta el resultado esperado en materias de construcción, apropiación de significados y formación de públicos.

Finalmente, sería valioso realizar estudios que consideren la opinión de los niños como promotores de la construcción de sentidos y valores a partir de un trabajo colaborativo entre productores e instituciones educativas, y desde allí, fomentar la alfabetización de medios (Aguaded, 2005; Fedorov, 2014; Pérez Tornero et al., 2015), en los que los infantes comenten las necesidades que tienen como televidentes y participen de forma activa en la producción de estas prácticas culturales que deriven en su formación social e identitaria a través de la proximidad de los contenidos educativos que emiten los canales de la televisión pública en Chile, Colombia y México, y no tanto por la televisión norteamericana. De esta manera, como lo menciona Livingstone (2005), los infantes pasarían de ser audiencias pasivas a conformar un público activo con un rol protagónico como coproductores y receptores de los contenidos emitidos a través de las pantallas.

\section{Referencias}

Aguaded, J. I. (2005). Estrategias de edu comunicacion en la sociedad audiovisual. Revista Comunicar, 24, 25-34.

Almada, M. (2014). Fuimos colores. Canal 22.

Ávila, J., y Acosta, C. (2016). Epistemología del pensamiento visual contemporáneo desde el imaginario transdisciplinario. Anagramas, 14(28), 167-240.

Buzeta, C., y Moyano, P. (2013). La medición de las audiencias de televisión en la era digital. Cuadernos.Info, 33, 53-62. https://doi.org/10.7764/cdi.33.503

Cabrera, L., y Veneras, V. (2013). Mr. Trance. Señal Colombia.

Cáceres, M. D., y Brändle, G. (2011). El uso de la televisión en un contexto multipantallas: viejas prácticas en nuevos medios. Análisi, 43, 21-44. 
Cahm, A., Kalagian, T., y Lyon, C. (2008). Business Models for Children's Media. En S. L. Calvert y B. J. Wilson (eds.), The Handbook of Children, Media, and Development (pp. 27 48). Blackwell Publishing. https://doi.org/10.1002/9781444302752

Ceppi, Á. y Covarrubias, H. (2016). Puerto papel. Chile, Brasil, Colombia E Argentina. TVN, Señal Colombia, Gloob \& Pakapaka.

Crespo-Pereira, V., Martínez-Fernández, V., y Campos-Freire, F. (2017). Neuroscience for Content Innovation on European Public Service Broadcasters. Comunicar, 52, 09-18. https://doi. org/10.3916/C52-2017-01

Díaz, Á., y Peirano, P. (2014). 31 minutos. TVN.

Egaña, A., y Puga, P. (2010). Tikitiklip. TVN.

Esomar (2009). Global Guidelines on Out-of-Home Audience Measurement. Version 1.0. Esomar Recuperado de https:/www.esomar.org/uploads/public/knowledge-and-standards/codes-and-guidelines/ ESOMAR_Global-Guidelines-on-Out-of-Home-Audience-Measurement_v.1.0.pdf

Federal Communications Commission (FCC) (2016). The V-Chip: Options to Restrict What Your Children Watch on TV. Consumer Guide. FCC. Recuperado de https:/www.fcc.gov/consumers/ guides/v-chip-putting-restrictions-what-your-children-watch

Fedorov, A. (2014). Media Education Literacy in the World: Trends. European Researcher, 67(1-2), 176-187. https://doi.org/10.13187/issn.2219-8229

Fernandes, T., y Remelhe, P. (2016). How to engage customers in co-creation: customers' motivations for collaborative innovation. Journal of Strategic Marketing, 24(3-4), 31 1 326. https:// doi.org/10.1080/0965254X.2015.1095220

Gómez, P. (2010). ¿Con qué sueñas? TVN.

González, C. (2010). La lleva. Ministerio de Cultura, RTVC Señal Colombia, Pontificia Universidad Javeriana y Canal 13.

Gozansky, Y. (2017). From pupils to consumers: the transformation of the concept of childhood in Israeli children's television. Journal of Children and Media, 11(1), 36-52. https://doi.org/10.1 080/17482798.2016.1203809

Hadas, L., y Shifman, L. (2013). Keeping the Elite Powerless: Fan-Producer Relations in the "Nu Who" (and New YOU) Era. Critical Studies in Media Communication, 30(4), 275-291. https://doi. org/10.1080/15295036.2012.676193

Hall, S. (2012). Encoding/Decoding. En M. Durham y D. Kellner (Eds.), Media and Cultural Studies: Keywords (2० ed, pp. 137-144). John Wiley E Sons.

Hallvard, M., Poell, T., y van Dijck, J. (2015). Rearticulating Audience Engagement: Social Media and Television. Television \& New Media, 17(2), 99-107. https://doi.org/10.1177/1527476415616194

Hartley, J. (2002). Cultural and Media Studies: The Key Concepts (Third Edit). Routledge. 
Livingstone, S. (2005). On the relation between audiences and publics. En S. Livingstone (Ed.), Audiences and publics: when cultural engagement matters for the public sphere (Vol. 2, pp. 17-42). Cultural Studies Intellect. https://doi.org/10.1177/02673231080230030504

Livingstone, S., y Das, R. (2013). The end of audiences? En J. Hartley, J. Burgess y A. Bruns (eds.), A Companion to New Media Dynamics (pp. 104-121). Blackwell Publishing. https://doi. org/10.1002/9781118321607

Martínez, C. (2016). "They Are Totally Unfiltered": Constructions of the Child Audience among Swedish Advertising Producers. Television \& New Media, 1-17. https://doi. org/10.1177/1527476416637676

Martínez, T. (2016). Mi lugar. Once Niños.

Mayer, V. (2016). The Places Where Audience Studies and Production Studies Meet. Television E New Media, 1 13. http://doi.org/10.1177/1527476416652482

Ortega, F., González Ispierto, B., y Pérez Peláez, M. E. (2015). Audiencias en revolución, usos y consumos de las aplicaciones de los medios de comunicación en tabletas y teléfonos inteligentes. Revista Latina de Comunicacion Social, 70, 627-651. https://doi.org/10.4185/RLCS$2015-1063$

Ortega, F., Pereira, C., e Igartua, J. J. (2016). A quantitative approach to the television programs aimed to child and youth audience in Brazil. Communication \& Society, 29(3), 49-67. https://doi. org/10.15581/003.29.3.49-68

Pearce, L. J., y Field, A. P. (2016). The Impact of "Scary" TV and Film on Children's Internalizing Emotions: A Meta-Analysis. Human Communication Research, 42(1), 98-121. https://doi. org/10.1111/hcre.12069

Pérez Tornero, J., Martínez-Cerdá, J. F., Portalés Oliva, M., Durán Becerra, T., Peralta García, L. y Julià Cano, A. (2015). Showing films and other audiovisual content in European Schools Obstacles and best practices. Digital Agenda for Europe. https://doi.org/10.2759/038024

Piotrowski, J. T. (2014). Participatory Cues and Program Familiarity Predict Young Children's Learning From Educational Television. Media Psychology, 17(3), 311-331. https://doi.org/10.1 080/15213269.2014.932288

Rincón, M., Rincón, M., y de Jesús, U. (2010). Guillermina y Candelario. Señal Colombia.

Salazar, O. (2012). Kiplata. Canal Once.

Sánchez, M. (2013). El mundo animal de Max Rodríguez. Señal Colombia, Pakapaka, Tribu 70 y Centro Ático.

Santamaría, Y. (2013). Camusi camusi. Señal Colombia.

Sekarasih, L., Nayar, K., O'Malley, D., Olson, C., y Scharrer, E. (2016). Entertaining audiences, ensuring inclusivity, and considering media influence: Sixth graders' understanding of media producers' responsibility. The Communication Review, 19(2), 128 152. https://doi.org/10.1080/1 0714421.2016 .1161338 
Vega, J., y Lafaurie, A. (2013). Observar TV: Un observatorio infantil de televisión para la interlocución de los niños. Revista Comunicar, 20(40), 145 153. https://doi.org/10.3916/C40 2013-03-05

Veloso, P. (2015). Pichintún. Chile: CNTV, Novasur.

Zanker, R. (2011). Producers Speak: Creating Civic Spaces for New Zealand Children. Media International Australia, (139), 32-41. 